IBIMA Publishing

Journal of Research and Practice in Dentistry

http://www.ibimapublishing.com/journals/DENT/dent.html

Vol. 2014 (2014), Article ID 959636, 6 pages

DOI: $10.5171 / 2014.959636$

Research Article

\title{
The Unusual Peg Shaped Mandibular Central Incisor - Report of Two Cases
}

\author{
Suchetha N Malleshi ${ }^{1}$, Srisha Basappa ${ }^{2}$, Shanta Negi ${ }^{3}$, A. Irshad ${ }^{4}$ \\ and Soumya K Nair ${ }^{5}$ \\ ${ }^{1}$ Department of Oral Medicine and Radiology, JSS Dental College and Hospital, JSS University, SS \\ Nagar, Mysore, India \\ ${ }^{2}$ Department of Oral Medicine and Radiology, Farooqia Dental College and Hospital, Umar Khayam \\ Road, Tilak Nagar, Mysore, India \\ ${ }^{3}$ Vasan Dental Care, Bangalore \\ ${ }^{4}$ Sparkle Dental Clinic, Tirupur, India \\ ${ }^{5}$ Om Dental Clinic, Mysore, India
}

Correspondence should be addressed to: Suchetha N Malleshi; smalleshi@yahoo.com

Received Date: 16 January 2014; Accepted Date: 17 February 2014; Published Date: 31 March 2014

Academic Editor: Tetsutaro Yamaguchi

Copyright (C) 2014 Suchetha N Malleshi, Srisha Basappa, Shanta Negi, A. Irshad and Soumya K Nair. Distributed under Creative Commons CC-BY 3.0

\begin{abstract}
Diverse morphological, structural and tooth number aberrations can occur within human dentition secondary to genetic and epigenetic influences. These deviations can manifest as microdontia, macrodontia, hypodontia, oligodontia to name a few. Non-syndromic microdontia is rarely noted, especially in the permanent mandibular incisors. This case report records two such rare cases of peg shaped microdont mandibular central incisor. In one case microdontia occurred synchronously with hypodontia. The facts about these malformations collectively form a body of knowledge with which would serve as a data base in monitoring the evolutionary process of human dentition.
\end{abstract}

Keywords: Microdontia, peg shaped teeth, mandibular central incisor, hypodontia.

\section{Introduction}

The human dentition is influenced by an array of multifarious, multilevel and multidimensional interactions between genetic, epigenetic and environmental factors. Brook (2009) stated that any mismatch between the molecular and cellular components during dental development manifests as distinct dental anomalies. These abnormalities may become apparent as structural and morphological variations or as alterations in the number of teeth. To name a few, the peculiarities may vary from microdontia, macrodontia, accessory cusps to hypodontia and hyperdontia. Permanent mandibular central incisor is rarely 
affected by tooth shape anomalies of crown and root (Nagaveni et al., 2011). Two rare cases of non syndromic peg shaped mandibular central incisor is communicated in this report.

\section{Case Report 1}

An apparently healthy 20 year old male reported for treatment of misaligned teeth. His dental, medical, family and personal history was non contributory. Intraoral examination revealed bilaterally over retained deciduous second molar and canine, palatal placed and rotated canines and clinically missing right second premolar in the maxillary arch. Also noted in the mandibular segment was the peg shaped right central incisor, clinically missing left lateral incisor and over retained left deciduous canine (Fig 1). The mandibular peg incisor was neither pulpally nor periodontally compromised. History revealed that the deciduous incisors were of normal morphology. Orthodontic evaluation revealed a Class I antero-posterior relationship with normal vertical proportions.

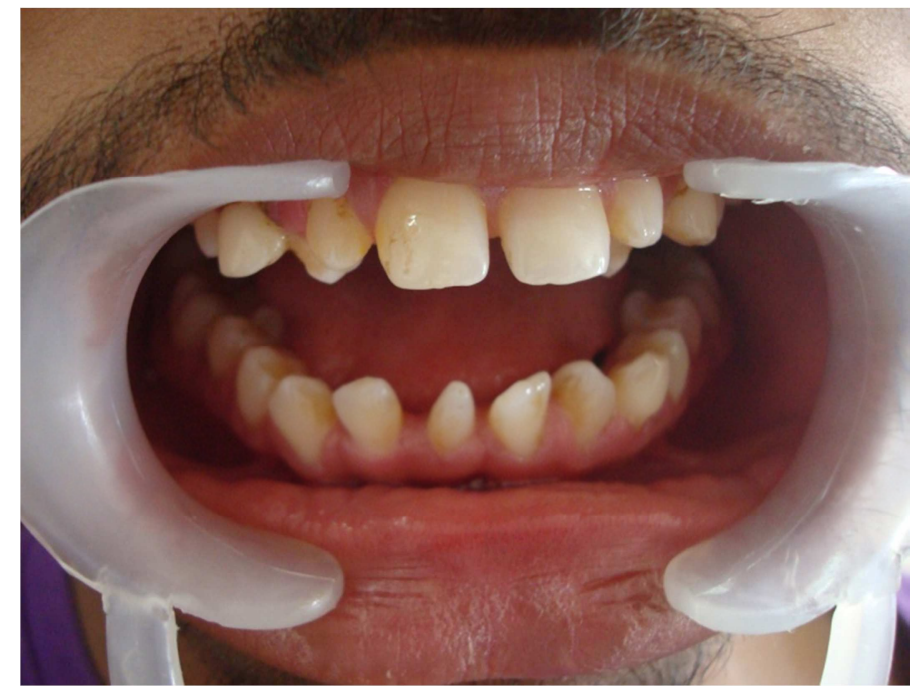

Figure 1: Peg Shaped Right Mandibular Central Incisor

On the panoramic radiography except for missing mandibular left lateral incisor, full compliment of permanent dentition including developing third molars was noted (Fig 2). IOPAR of the conical shaped mandibular central incisor revealed normal root length with no evidence of any high pulp horn or pulp stones.

A diagnosis of non-syndromic occurrence of peg mandibular central incisor with hypodontia was arrived at. The patient remains under review for orthodontic treatment and aesthetic rehabilitation.

\section{Case Report 2}

A 9 year old boy in mixed dentition stage reported for treatment of pain of pulpal origin in the decayed deciduous maxillary right first molar. His dental, medical, family and personal history was non contributory. Dental examination revealed multiple carious teeth. Also conspicuous was the peg shaped permanent mandibular left central incisor (Fig 3). No other dental morphologic or skeletal abnormalities were appreciable. History about the deciduous mandibular incisors suggested that they were of normal morphology and were not subjected to any trauma. Mandibular anterior occlusal radiograph did not show any pulp stones, rhizomicry or periapical changes in the peg mandibular central (Fig 4). 


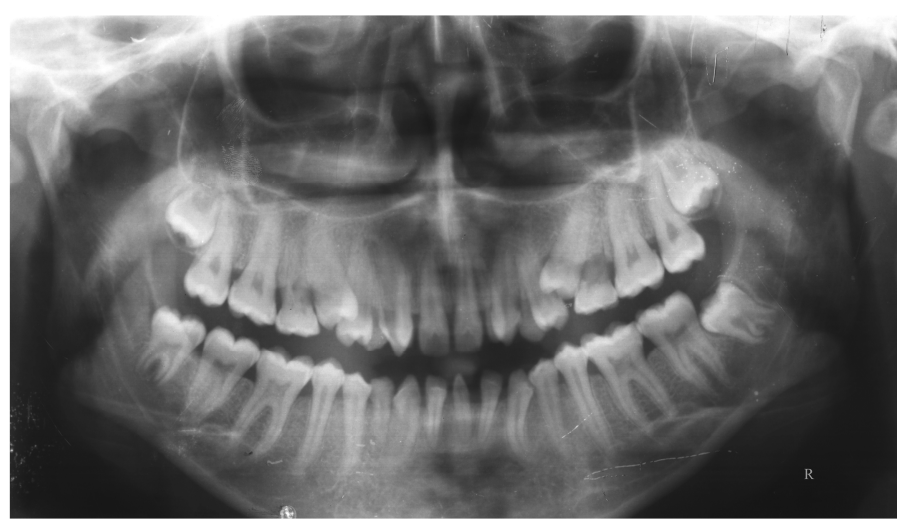

Figure 2: Cropped Panoramic Radiograph Displaying the Peg Microdontia and Hypodontia

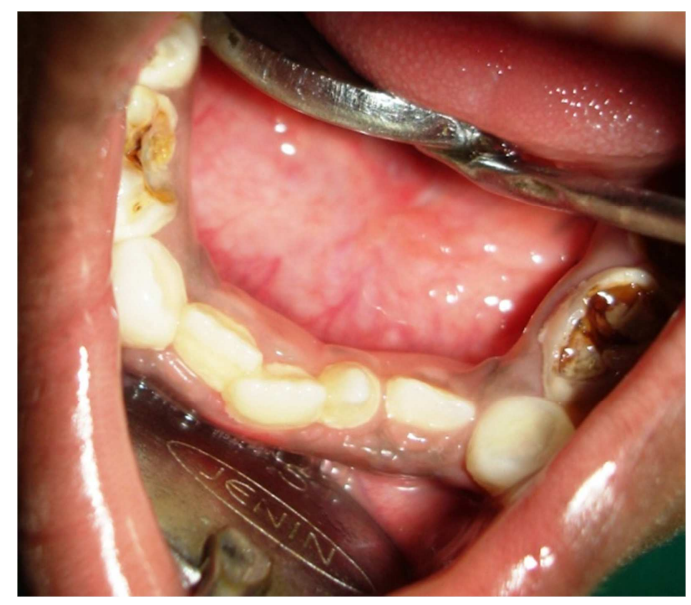

Fig 3: Occlusal Clinical View of the Peg Shaped Mandibular Left Central Incisor

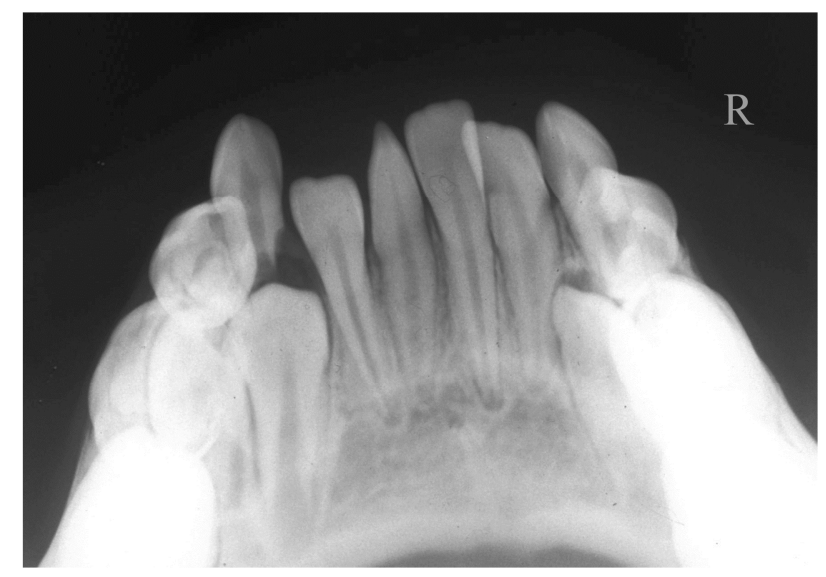

Figure 4: Mandibular Anterior Occlusal Radiograph Demonstrating the Peg Central

As with above case a diagnosis of nonsyndromic occurrence of peg mandibular central incisor was arrived at. The treatment for presenting complaint and other decayed teeth were initiated and patient is under review for a phased conservative and orthodontic treatment. 


\section{Discussion}

Genetic and epigenetic factors regulate the occurrence of a variety of tooth morphological aberrations. Environmental factors like cytotoxic medications, trauma, radiation or pulpal complications during development can also be contributory to the genesis of these malformations (Koch et al., 2009). A sequence of reciprocal communications between ectodermal and mesenchymal factors regulates, the initiation i.e. tooth region and number, the morphogenesis i.e. tooth type, size, shape including dimensions and cusp number and the differentiation i.e. tooth structure enamel and dentine formation and mineralization (Brook, 2009).

Altered morphodifferentiation results in tooth size variations. Koch et al., (2009) defined tooth size as abnormal, when dimensions deviate two standard deviations from average. ${ }^{3}$ This size abnormality can manifest either as macrodontia or microdontia. Microdontia is a rare phenomenon. The term microdontia (microdentism, microdontism) designates the condition displaying abnormally small teeth. As defined by Kaplan microdont is a small tooth with greater than 3.5 standard deviation below the sex-specific mean mesio-distal tooth size (Anziani et al., 2010). According to Neville et al., (2009) tooth size variation exists also between races and genders.

Microdontia is chiefly divided into three types (Neville et al., 2009, Shafer 1993):

(1) True generalized microdontia, in which all the teeth are smaller than normal, is exceedingly rare; can be seen in pituitary dwarfism, Down syndrome and various other hereditary disorders.

(2) Relative generalized microdontia - that is normal sized teeth appearing relatively small due to macrognathia

and

(3) Microdontia involving only a single tooth.
Bargale et al., (2011) classified microdontia of a single tooth as: ${ }^{7}$

(1) microdontia of the whole tooth,

(2) microdontia of the crown of the tooth,

and

(3) microdontia of the root alone.

Microdontia can encompass different tooth contours and morphologies. The most cited example of localized microdontia, is of maxillary lateral incisors - called "peg lateral". Peg shaped teeth are anterior teeth in the primary or permanent dentition with a crown diameter that decreases markedly from cervical margin to incisal edge, thus resulting in the characteristic peg shape. The reported prevalence varies from 0.8$8.4 \%$ of the population. After maxillary lateral incisor, the third molars are the ones to be commonly noted microdonts (Anziani et al., 2010and Neville et al., 2009). An extensive review of English literature yielded only three published cases of isolated non syndromic peg shaped permanent mandibular central incisor, thus making it a rarity (Anziani et al., 2010, Ramachandra et al., 2009, Chanchala et al., 2012). Cases of non syndromic generalized microdontia has also been reported (Bargale et al., 2011, Nagaveni et al., 2011, Nagaveni 2012, Sharma 2001, Ather et al., 2013). Altug - Atac et al., 2007 reported two cases of mandibular peg shaped lateral incisors.

The syndromes associated with microdontia are Gorlin-Chaudhry-Moss syndrome, Williams's syndrome, UllrichTurner syndrome, Chromosome 13, Rothmund-Thomson syndrome, Hallermann-Streiff, Orofaciodigital syndrome (type 3), Oculo-mandibulo-facial syndrome, Tricho-Rhino-Phalangeal and type1 Branchiooculo-facial syndrome (Bargale et al., 2011).

Shafer et al., 1993 has stated that microdontia shows s strongly associated with hypodontia. Antunes et al., 2013 suggested that tooth agenesis and pegshaped or strongly mesio-distally reduced 
upper lateral incisors could possibly have the same genetic background. Hypodontia, the most common developmental dental anomaly in humans has a prevalence of $1.6 \%$ to $13.3 \%$ in diverse populations. World Health Organization regards the congenital absence of permanent teeth as a handicapping dentofacial anomaly (Anziani et al., 2010).

The term hypodontia refers to the congenital agenesis of one or more (up to six) teeth except third molars. When this number (excluding third molars) exceeds six, the condition is designated as oligodontia, with anodontia denoting complete absence of teeth (Anziani et al., 2010, Ramazanzadeh et al., 2013).

In both hypodontia and microdontia, the prevalence varies between studies and racial groups. Hypodontia shows a female preponderance (Anziani et al., 2010). Tooth agenesis can just an isolated finding or part of a syndrome. In addition to inherited defects, jaw fractures, surgical procedures, chemotherapy, cranial irradiation early in development, somatic diseases such as syphilis, scarlet fever, rickets, nutritional disturbances during pregnancy or infancy or can affect tooth development (Antunes et al., 2013, Vastardis, 2007). Hypodontia is frequently associated with altered craniofacial morphology and dental abnormalities such as cleft, palatally dislocated canines, disturbed exfoliation and eruption, infraocclusion of primary molars, enamel hypoplasia, taurodontism and abnormal contour and size of maxillary lateral incisors. Although controversy exists about association of tooth size in patients with congenitally missing teeth, ample evidence exists for coexistence of hypodontia with microdontia (Anziani et al., 2010, Altug - Atac et al., 2007).

Tooth morphology and number variations are usually noticed but rarely acknowledged. Documentation of these discrepancies helps in assessing the evolutionary structural and morphological changes in human dentition. The present case report highlights the association between microdontia and hypodontia, which can affect management and treatment planning. Since, dental esthetics is known to affect the overall quality of life, it is important that a multidisciplinary approach is adopted in the treatment of patients with tooth agenesis and malformation.

\section{References}

Altug-Atac A. T. \& Erdem, D. (2007). "Prevalence and Distribution of Dental Anomalies in Orthodontic Patients," American Journal of Orthodontics and Dentofacial Orthopedics, 131(4) 510-4.

Antunes, L. A. A., Küchler, E. C., Costa, M. C., Antunes, L. A. \& Granjeiro, J. M. (2013). "Discordant Tooth Agenesis and Peg Shaped in a Pair of Monozygotic Twins: Clinical and Molecular Study," Dental Research Journal (Isfahan), 10(6) 820-824.

Anziani, H., Cole, B. \& Hobson, R. (2010). "An Unusual Dental Anomaly in a Hypodontia Patient," Dental Update, 37(10) 691-5.

Ather, A., Ather, H., Acharya, S. R. \& Radhakrishnan, R. A. (2013). "Lobodontia: The Unravelling of the Wolf Teeth," Romanian Journal of Morphology and Embryology, 54(1) 215-7.

Bargale, S. D. \& Kiran, S. D. P. (2011). "NonSyndromic Occurrence of True Generalized Microdontia with Mandibular Mesiodens A Rare Case," Head \& Face Medicine, 7:19.

Brook, A. H. (2009). "Multilevel Complex Interactions between Genetic, Epigenetic and Environmental Factors in the Aetiology of Anomalies of Dental Development," Archives of Oral Biology, 54 Suppl 1:S3-17.

Chanchala, H. P. \& Nandlal, B. (2012). "Coexistent Peg Shaped Mandibular Central Incisors Along with Maxillary Lateral Incisors: A Rare Case," International Journal of Oral \& Maxillofacial Pathology, 3(1):6568.

Koch, G. \& Poulsen, S. (2009). Pediatric Dentistry: A Clinical Approach. 2nd Edition. Blackwell Publishing Ltd. United Kingdom. 
Nagaveni, N. B. (2012). "An Unusual Occurrence of Multiple Dental Anomalies in a Single Nonsyndromic Patient: A Case Report," Case Reports in Dentistry.

Nagaveni, N. B., Umashanikara, K. V., Vidyullatha, B. G., Sreedevi, S. \& Radhika, N. B. (2011). "Permanent Mandibular Incisor with Multiple Anomalies - Report of a Rare Clinical Case," Brazilian Dental Journal, 22(4):346-50.

Neville, B. W., Damm, D. D., Allen, C. M. \& Bouquot, J. E. (2009). Oral and Maxillofacial Pathology. 3rd Ed. India: Saunders.

Ramachandra, S. S., Baliga, V. \& Jithendra, K. D. (2009). "Peg Shaped Mandibular Central Incisor," Dental Update, 36(7):439 - 441.
Ramazanzadeh, B. A., Ahrari, F. \& Hajian, S. (2013). "Evaluation of Tooth Size in Patients with Congenitally-Missing Teeth," Journal of Dental Research, Dental Clinics, Dental Prospects, 7(1):36-41.

Shafer, W. G., Hine, M. K., Levy, B. M. \& Tomich, C. E. (1993). 'Text Book of Oral Pathology,' 4th Ed. Philadelphia: Saunders.

Sharma, A. (2001). "Unusual Localized Microdontia: Case Reports," Journal of Indian Society of Pedodontics and Preventive Dentistry, 19(1): 38 -9.

Vastardis, H. (2000). "The Genetics of Human Tooth Agenesis: New Discoveries for Understanding Dental Anomalies," American Journal of Orthodontics and Dentofacial Orthopedics, 117(6):650-6. 